\title{
Distinctive Patterns of Tau Accumulation and White-Matter Degeneration on Domain- Specific Neuropsychiatric Test Scores
}

\author{
Zack Hall ${ }^{1}$, Billy Chien ${ }^{1}$, Shannon L Risacher ${ }^{2}$, Andrew J Saykin², Yu-Chien Wu², Qiuting Wen² \\ ${ }^{1}$ Indiana University School of Medicine, ${ }^{2}$ Indiana University School of Medicine, department of \\ Radiology \& Imaging Science
}

\section{Background:}

Diagnosis of $A D$ is often started via cognitive tests such as the Mini Mental Status Exam (MMSE) or Montreal Cognitive Assessment (MOCA) with the diagnosis being finalized with autopsy. Currently, positron emission tomography (PET) tracers are used to visualize tau and beta amyloid accumulation in the brain. These imaging techniques demonstrate significant correlations with cognitive decline and have been used to aid in the diagnosis of AD.

Particularly, tau accumulation has been shown to progress in a distinct pattern and has been linked to white matter degeneration. We hypothesized that tau accumulation and white matter degeneration in different brain regions have unique correlation patterns with domain-specific neuropsychiatric test scores.

\section{Methods:}

This study included 87 older adults ( 57 cognitively normal subjects and 27 mild cognitive impairment) from the Indiana Alzheimer's Disease Center. All participants underwent tau-PET ([18F] Flortaucipir PET) and diffusion MRI (dMRI) exams. Tau accumulation was quantified with tau-PET SUVR. White-matter structural connectivity (SC), which probes the integrity of whitematter connections of the brain, was quantified by metrices extracted from dMRI using network analysis. Both tau accumulation and SC measurements were quantified in 84 cortical region of interests (ROIs) and were compared to their domain specific neuropsychiatric test scores with linear regression analysis.

\section{Results:}

The verbal memory, visual memory, and visuospatial ability all had unique region-specific correlations with white-matter degeneration and tau accumulation. Verbal memory was solely correlated with tau accumulation. Visual memory was related to both tau accumulation and white-matter SC. Finally, visuospatial ability was only correlated with white-matter SC.

\section{Potential Impact:}

This data reveals that although there is a tight interplay between tau accumulation and whitematter degeneration, they affect brain functions in different ways. Specifically, grey-matter tau accumulation is associated with overall memory loss and decrease in the white-matter connectivity affects the visual-related information processing. 